\title{
Beaud Stéphane, La France des Belhoumi. Portraits de famille (1977-2017)
}

\section{Maryse Tripier}

\section{(2) OpenEdition \\ 1 Journals}

Édition électronique

URL : https://journals.openedition.org/remi/12190

DOI : $10.4000 /$ remi. 12190

ISSN : 1777-5418

Éditeur

Université de Poitiers

\section{Édition imprimée}

Date de publication : 1 décembre 2018

Pagination : 234-237

ISBN : 979-10-90426-63-4

ISSN : 0765-0752

Référence électronique

Maryse Tripier, « Beaud Stéphane, La France des Belhoumi. Portraits de famille (1977-2017) », Revue européenne des migrations internationales [En ligne], vol. 34 - n4 | 2018, mis en ligne le 28 décembre 2018, consulté le 15 avril 2022. URL : http://journals.openedition.org/remi/12190 ; DOI : https:// doi.org/10.4000/remi. 12190

Ce document a été généré automatiquement le 15 avril 2022.

(c) Université de Poitiers 


\title{
Beaud Stéphane, La France des Belhoumi. Portraits de famille (1977-2017)
}

\author{
Maryse Tripier
}

\section{RÉFÉRENCE}

Beaud Stéphane (2018) La France des Belhoumi. Portraits de famille (1977-2017), Paris, La

Découverte, 352 p. , ISBN : 978-2-36935-089-7

1 Cet ouvrage ${ }^{1}$ est le produit d'une longue et vaste enquête qui retrace le destin social et professionnel, des huit enfants (cinq filles, trois garçons) d'une famille algérienne installée en France depuis 1977, dans un quartier HLM d'une petite ville de province. Y sont pointés les obstacles et les ressources vers une intégration que l'auteur qualifie de « silencieuse ». Cette enquête n'est pas « sur contrat », mais "par contrat » avec la fille aînée de cette famille, Samira ${ }^{2}$. Elle a permis à Stéphane Beaud de rencontrer physiquement, téléphoniquement, par courrier et e-mails, les parents et les huit enfants de cette fratrie issue de l'immigration algérienne. L'enquête est longue, de 2012 à 2017, et engage émotionnellement et éthiquement le sociologue, qui discute aussi avec les intéressées de ses interprétations en cours d'enquête. Spécialiste de la classe ouvrière, des jeunes des quartiers populaires, "sociologue d'enquête " affirmé, cet ouvrage prolonge les travaux antérieurs de Stéphane Beaud, avec une plus grande ampleur (Beaud, 2014).

L'univers ici décrit, le rapport à «l'objet» devenu sujet, se situe dans la ligne des travaux de Sayad (2006) sur les différents âges de l'immigration algérienne. Il s'agit du dernier âge, celui qui va nous interroger sur les « enfants illégitimes ». Alors que le plus souvent on parle d'eux, ou pour eux, ici ce sont d'abord eux qui parlent et nous donnent à voir et à comprendre leur vie. L'autre référence qui vient à l'esprit est la monographie de Lewis (1978) Les enfants de Sanchez. Monographie d'une famille pauvre 
qui émigre de la campagne vers Mexico. Même attention à chaque enfant et même empathie. Si Lewis en a théorisé une "culture de la pauvreté ", ici on s'intéresse à l'intégration, au mouvement. L'avantage de cette monographie est de tisser à l'échelle de l'individu et de la famille, les liens entre des "variables", des dimensions habituellement traitées séparément: l'échec/la réussite scolaire, l'insertion professionnelle, la mobilité résidentielle, les stratégies matrimoniales, le rapport au politique où à la religion. Ces données segmentées nous sont livrées le plus souvent, à l'échelle statistique nationale, et le changement n'est alors perceptible que par des panels réguliers. Pour autant, l'auteur ne voit aucune concurrence entre ces approches. Il considère que ses résultats corroborent le plus souvent l'enquête TEO qui travaille sur le destin des groupes minoritaires (Beauchemin et al., 2016).

3 Le temps, donc le changement social, est ici traité dans l'enquête elle-même, puisqu'au sein de la fratrie, un écart de seize ans sépare les aînées des cadets (cadettes). C'est ainsi que Stéphane Beaud qualifie les sœurs aînées «d'héritières de la marche pour l'Égalité de 1983 », et retrace les espoirs nés de l'élection de François Mitterrand. Par contraste les trois cadettes se retrouvent dans l'orbite de la "génération des cités ». Dans, selon ses propres termes, « une France moins bienveillante et face à une gauche qui a failli ». S'il y a un groupe des enfants de l'immigration algérienne, voire maghrébine, il est hétérogène et traversé de nombreux clivages ici décrits.

4 Le temps est aussi celui de l'enquête elle-même, car les attentats de 2015, changent la donne pour les familles issues de l'immigration maghrébine, pour les «arabes", qui doivent se prononcer et d'une certaine manière s'identifier : "Charlie/pas Charlie » par exemple. L'objectif premier de cet ouvrage est donc de déconstruire une image stéréotypée des jeunes issus de l'immigration algérienne, symboles de l'échec supposé de l'intégration. En effet un des apports majeurs de ce livre est de montrer des parcours d'intégration "silencieuse », de nommer les obstacles et les ressources qu'il a fallu mobiliser pour y parvenir.

Ce faisant il a une fonction miroir sur la société française. Le contexte de scolarisation des sœurs aînées, la mobilisation de certaines institutrices, l'offre de la municipalité communiste de l'époque ont aidé les deux sœurs aînées (fortement déterminées et courageuses) à réussir leur parcours scolaire, le prolonger et à accéder à la mobilité géographique et à l'insertion dans les classes moyennes. S'agissant du rôle des aînés, il est à double tranchant, mais très prédictif. Ici les aînées donnent les clés du succès, soutiennent les cadets, mais si a contrario, comme nous l'avons observé, les aînés sont mauvais élèves, perturbateurs ou décrocheurs, l'effet de réputation rejaillit sur la fratrie et discrédite les parents (Léger et Tripier, 1986). Les municipalités de banlieue se plaignent que ceux qui réussissent quittent leur quartier et qu'elles accueillent en lieu et place des familles dont la vie est difficile. Même constat dans les écoles, départ à zéro à nouveau, cercle vicieux. Ce livre fait un constat important $:$ il a fallu déjà partir pour réussir. Mobilité sociale, géographique et professionnelle s'entrecroisent. Partir c'est aussi acquérir de la liberté face à l'injonction au mariage pour les filles, au poids du « qu'en dira-t-on ». En fin d'ouvrage, on voit le sort qui est réservé à ceux et celles qui restent sans ressources matérielles et capital scolaire. Les femmes trouvent un statut dans la maternité, le poids du contrôle social à dominante religieuse s'accroît. Le contexte a changé, moins de soutien des enseignants, désertification des services publics, stigmatisation accrue depuis les attentats de 2015, poids croissant des « barbus » (Chemin et Bacqué, 20183). 
6 Néanmoins la solidarité affective et matérielle est maintenue même à distance entre la Région parisienne et la petite ville d'origine. Les liens existent avec l'Algérie, mais sont moins prégnants. Cette solidarité matérielle rappelle que les Belhoumi sont pauvres et connaissent de grandes difficultés matérielles. Ce qui marque leur appartenance première au monde ouvrier et populaire. En ce sens ils nous parlent de toutes les familles qui ont du mal à joindre les deux bouts tout en tentant d'échapper à l'assistanat. L'apport sociologique est qu'il faut traiter des familles et non des individus, c'est l'unité pertinente, particulièrement dans l'immigration. Le père de cette famille est en invalidité très jeune. Si beaucoup de travaux ont montré l'importance des accidents du travail sur les pères, encore trop peu ont mis en relation la fin de carrière des OS et les effets de leur désocialisation. Chibanis dans les foyers, vieillissant seuls, ou retour au pays sans savoir-faire à faire valoir par exemple. Ainsi à Billancourt, et dans l'automobile, les "OS à vie" n'ont pu transmettre, ni métier, ni réseau, ni codes permettant à leurs enfants d'accéder aux stages qualifiants. Fini le Comité d'entreprise, les colonies de vacances, le bibliobus, etc. Le rôle de l'environnement local et de l'École devient alors central. Si l'embauche des enfants du personnel a encore quelque persistance, on la retrouvera à la Poste, la RATP, la SNCF, mais plus dans l'industrie, l'intérim y est anonyme.

7 S'il faut parler du style, ce livre se lit comme un roman. Loin de la focalisation médiatique sur les jeunes dits «musulmans », voire radicalisés, cet ouvrage nous livre des portraits qui donnent aux enquêtée's un quasi-statut de personnage d'une saga. Samira, Leila, Rachid, Azzedine, Mounir, Dalila, Amel, Nadia. On a l'impression de les connaître après avoir suivi leurs parcours, toujours difficiles, parfois chaotiques, mais qui signent leur entrée dans le « club France». Cet ouvrage est à mettre entre toutes les mains, au-delà des sociologues et des chercheurs sur l'immigration.

\section{BIBLIOGRAPHIE}

Beauchemin Cris, Hamel Christelle et Simon Patrick (Dirs.) (2016) Trajectoires et origines. Enquête sur la diversité des populations de France, Paris, INED, 624 p.

Beaud Stéphane (2014) Affreux, riches et méchants? Un autre regard sur les Bleus, Paris, La Découverte, $280 \mathrm{p}$.

Chemin Ariane et Bacqué Raphaëlle (2018) La communauté, Paris, Albin Michel, 336 p.

Léger Alain et Tripier Maryse (1986) Fuir ou construire l'École populaire, Paris, Méridiens Klincksiek, $206 \mathrm{p}$.

Lewis Oscar (1978 [1961]) Les Enfants de Sanchez. Autobiographie d'une famille mexicaine, Paris, Gallimard, 644 p.

Sayad Abdelmalek (2006) L'immigration ou les paradoxes de l'altérité. 2. Les enfants illégitimes, Paris, Raisons d'agir, $208 \mathrm{p}$. 


\section{NOTES}

1. L'auteure de cette note a consacré une grande part de son activité scientifique aux travailleurs immigrés en France dans la période considérée et a échangé avec Stéphane Beaud sur l'apport de son enquête.

2. Les noms des lieux et des personnes ont été changés.

3. Dans cet ouvrage de Chemin et Bacqué sur la ville de Trappes, on retrouve bien des processus décrits dans le livre de Stéphane Beaud.

\section{AUTEURS}

\section{MARYSE TRIPIER}

Professeure émérite, Urmis/Université Paris Diderot 